\title{
On mountain pass theorem and its application to periodic solutions of some nonlinear discrete systems
}

\section{Liang Ding ${ }^{1,2}$, Jinlong Wei ${ }^{3^{*}}$ (D) and Shiqing Zhang ${ }^{2}$}

"Correspondence:

weijinlong@zuel.edu.cn

${ }^{3}$ School of Statistics and

Mathematics, Zhongnan University

of Economics and Law, Wuhan,

China

Full list of author information is

available at the end of the article

\begin{abstract}
In this paper, we prove a new quantitative deformation lemma, and then gain a new mountain pass theorem in Hilbert spaces. By using the new mountain pass theorem, we obtain the new existence of two nontrivial periodic solutions for a class of nonlinear second-order discrete systems, which greatly improves the result in (Zhou et al. in Proc. R. Soc. Edinb. A 134:1013-1022, 2004).
\end{abstract}

MSC: Primary 49J35; secondary 34K13; 34G20

Keywords: Critical points; Quantitative deformation lemma; Mountain pass theorem; Nontrivial periodic solutions; Discrete systems

\section{Introduction and main results}

It is well known that the classical mountain pass theorem of Ambrosetti-Rabinowitz [2] has proved to be a powerful tool in applications of many areas to obtain the existence of periodic solutions. We first recall the famous theorem.

Theorem 1.1 ([2]) Let $X$ be a Banach space, $\varphi \in C^{1}(X, R)$. Suppose there exist $e \in X$ and two real numbers $\alpha>0$ and $r>0$ such that $\|e\|>r$ and

(i) $\varphi(u) \geq \alpha>0$ on $\{u \in X \mid\|u\| \leq r\} \backslash\{0\}$;

(ii) $\varphi(0)=\varphi(e)=0$;

(iii) if $\left(u_{n}\right) \subset X$ with $0<\varphi\left(u_{n}\right), \varphi\left(u_{n}\right)$ bounded above, and $\varphi^{\prime}\left(u_{n}\right) \rightarrow 0$, then $\left(u_{n}\right)$ possesses a convergent subsequence.

Then $c:=\inf _{\gamma \in \Gamma} \max _{t \in[0,1]} \varphi(\gamma(t))$, where

$$
\Gamma:=\{\gamma \in C([0,1], X): \gamma(0)=0, \gamma(1)=e\},
$$

is a critical value of $\varphi$.

Since then, there have been many variant generalizations for the above mountain pass theorem [3-5]. Elegant work was done by Willem [3]. When proving the mountain pass theorem, we should introduce a quantitative deformation lemma first (also see [6, 7]).

(c) The Author(s) 2019. This article is distributed under the terms of the Creative Commons Attribution 4.0 International License (http://creativecommons.org/licenses/by/4.0/), which permits unrestricted use, distribution, and reproduction in any medium, provided you give appropriate credit to the original author(s) and the source, provide a link to the Creative Commons license, and indicate if changes were made. 
Lemma 1.1 (Quantitative deformation lemma [3]) Let $X$ be a Hilbert space, $\varphi \in C^{2}(X, R)$, $c \in R, \varepsilon>0$. Assume that

$$
\left\|\varphi^{\prime}(u)\right\| \geq 2 \varepsilon, \quad \forall u \in \varphi^{-1}([c-2 \varepsilon, c+2 \varepsilon]) .
$$

Then there exists $\eta \in C(X, X)$, such that

(i) $\eta(u)=u, \forall u \notin \varphi^{-1}([c-2 \varepsilon, c+2 \varepsilon])$;

(ii) $\eta\left(\varphi^{c+\varepsilon}\right) \subset \varphi^{c-\varepsilon}$, where $\varphi^{c-\varepsilon}:=\varphi^{-1}((-\infty, c-\varepsilon])$.

Using Lemma 1.1, Willem established the following mountain pass type theorem.

Theorem 1.2 ([3]) Let $X$ be a Hilbert space, $\varphi \in C^{2}(X, R), \quad c_{0}>c_{1}, \quad c:=$ $\inf _{\gamma \in \Gamma} \max _{t \in[0,1]} \varphi(\gamma(t))$, where $c_{1}:=\max \{\varphi(0), \varphi(e)\}, c_{0}:=\inf _{\|u\|=r} \varphi(u)$ and

$$
\Gamma:=\{\gamma \in C([0,1], X): \gamma(0)=0, \gamma(1)=e\} .
$$

\section{Suppose}

(i) $\varphi$ satisfies the (P.S.) condition ( $\varphi$ is said to satisfy the (P.S.) condition, if any sequence $\left\{u^{(k)}\right\} \subset X$ satisfying $\varphi\left(u^{(k)}\right)$ is bounded and $\varphi^{\prime}\left(u^{(k)}\right) \rightarrow 0$ as $k \rightarrow+\infty$, possesses a convergent subsequence);

(ii) there exist $e \in X$ and $r>0$ be such that $\|e\|>r$ and $c_{0}>\varphi(0) \geq \varphi(e)$.

Then $c$ is a critical value of $\varphi$.

Let $c_{0}$ and $c_{1}$ be stated in Theorem 1.2. We see that Theorems 1.1 and 1.2 hold for $c_{0}>c_{1}$. A few years later, this condition is relaxed to $c_{0} \geq c_{1}$ in [8] (also see [9]):

Theorem 1.3 ([8]) Let $X$ be a Banach space and $X$ has finite dimension, $\varphi \in C^{1}(X, R)$. Suppose there exist $e \in X$ and two real numbers $a$ and $r>0$ such that $\|e\|>r$ and

(i) $c_{0} \geq a, \varphi(0) \leq a, \varphi(e) \leq a$;

(ii) any sequence $\left(u_{n}\right)$ in $X$ such that $\varphi\left(u_{n}\right) \rightarrow$ limit $\geq a$, and $\varphi^{\prime}\left(u_{n}\right) \rightarrow 0$ possesses a convergent subsequence.

Then $c$ is a critical value of $\varphi$.

Clearly, all above results are based upon the relationship between $c_{0}$ and $c_{1}$. Then an interesting question is raised: can we obtain a mountain pass type theorem, which is independent of $c_{0}$ ? In this paper, we give a positive answer and the mountain pass type theorem is given by:

Theorem 1.4 Let $X$ be a Hilbert space, $\varphi \in C^{2}(X, R), e, e_{1} \in X, r>0$ be such that $0<\left\|e_{1}\right\|<$ $r$ and $\|e\|>r$, and $\varphi(0)<\varphi(e)=\varphi\left(e_{1}\right)$. Then, for each small enough $\varepsilon>0$, there exists $\hat{u} \in X$ such that

(i) $\hat{c}-2 \varepsilon \leq \varphi(\hat{u}) \leq \hat{c}+2 \varepsilon$;

(ii) $\left\|\varphi^{\prime}(\hat{u})\right\|<2 \varepsilon$,

where $\hat{c}:=\inf _{\gamma \in \hat{\Gamma}} \max _{t \in[0,1]} \varphi(\gamma(t))$ and

$$
\hat{\Gamma}:=\left\{\gamma \in C([0,1], X): \gamma(0)=0, \gamma\left(\frac{1}{2}\right)=e_{1}, \gamma(1)=e\right\} .
$$


Remark 1.1 The new mountain pass theorem is independent of $c_{0}$, and if $\varphi$ satisfies the (P.S.) condition, there exists $\hat{u} \in X$ such that $\varphi(\hat{u})=\hat{c}$.

Now, we turn to an application of this mountain pass type theorem to the existence of periodic solutions of discrete systems, which have been appeared in computer science, economic, neural networks, ecology, cybernetics, etc. and which were extensively investigated in $[1,10-18]$.

Let $Z, N, R$ be the set of all integers, natural numbers and real numbers, respectively. In [13], by critical point theory, Guo and Yu established the existence of two nontrivial $M$-periodic solutions to the following discrete difference equations:

$$
\Delta^{2} u_{n-1}+f\left(n, u_{n}\right)=0, \quad n \in Z,
$$

where $u_{n}=u(n) \in R$ and

$$
f\left(n, u_{n}\right)=\nabla_{u_{n}} F\left(n, u_{n}\right), \quad \Delta u_{n}=u_{n+1}-u_{n}, \quad \Delta^{2} u_{n}=\Delta\left(\Delta u_{n}\right),
$$

and $F: Z \times R \rightarrow R, F(n, x)$ is continuously differentiable in $x$ for every $n \in Z$ and $T$-periodic $(0<T \in N)$ in $n$ for all $x \in R$.

If $u_{n} \not \equiv 0, \forall n \in Z$, is a $M$-periodic solution of system (1.1), then we call $u_{n}$ a nontrivial $M$ periodic solution of system (1.1). It is well known that [13] is one of the original papers to study the existence of nontrivial periodic solutions of system (1.1) for superlinear $f\left(n, u_{n}\right)$ at $u_{n}$ (also see $[12,17]$ ). When $f\left(n, u_{n}\right)$ is sublinear in the second variable $u_{n}$, we refer the reader to $[14,16]$, and for the case of $f\left(n, u_{n}\right)$ is neither superlinear nor sublinear, we refer to [1]. For more details in this direction, one consults to [10, 11, 15, 18]. It is remarked that, in [1], under the assumptions described below:

( $\left.\mathrm{A}_{1}\right) F \geq 0, F \in C^{1}(R \times R, R)$, and for every $(n, x) \in Z \times R$, there is a positive integer $M \geq 3$ such that $F(n+M, x)=F(n, x)$;

$\left(\mathrm{A}_{2}\right)$ there exist constants $\delta>0, \alpha \in\left(0,1-\cos \frac{2 \pi}{M}\right)$ such that

$$
F(n, x) \leq \alpha x^{2} \quad \text { for } n \in N, x \in R \text { and }|x| \leq \delta
$$

$\left(\mathrm{A}_{3}\right)$ there exist constants $w_{1}>0, w_{2}>0$ and $w_{3} \in(2,+\infty)$ when $M$ is even or $w_{3} \in(1+$ $\left.\cos \frac{\pi}{M},+\infty\right)$ when $M$ is odd, such that

$$
F(n, x) \geq w_{3} x^{2}-w_{2} \quad \text { for } n \in N,|x| \geq w_{1},
$$

by using the linking theorem in [15], Zhou, Yu and Guo derived the existence of two nontrivial $M$-periodic solutions for system (1.1), and they gave an example:

Example 1.1 Take $F(t, x)=a\left(x^{2} / 2+\cos x-1\right)(\phi(t)+K)$ with $x \in R$, constant $K>0$, where constant $a$ and positive integer $M \geq 3$ satisfy

$$
\begin{cases}a>2, & \text { when } M \text { is even, } \\ a>2\left(1+\cos \frac{\pi}{M}\right), & \text { when } M \text { is odd, }\end{cases}
$$

$\phi(t) \in C^{1}(R, R)$, and $\phi(t)$ is a $M$-periodic function satisfying $|\phi(t)|<K$. 
Obviously, by conditions $\left(\mathrm{A}_{1}\right)$ and $\left(\mathrm{A}_{2}\right)$, we deduce that $F(n, 0)=0, \forall n \in Z$. However, the opposite conclusion does not hold. So, it is also natural to ask: if $f\left(n, u_{n}\right)$ is neither superlinear nor sublinear such that $F(n, 0)=0, \forall n \in Z$, can we still obtain the existence of two nontrivial periodic solutions?

Moreover, in the original paper [13], we note that $F \in R$ but not $F \geq 0$. So, another interesting question is raised: for the case of $f\left(n, u_{n}\right)$ is neither superlinear nor sublinear at $u_{n}$, if we omit this nonnegative restriction on $F$, can we still obtain two nontrivial $M$-periodic solutions for system (1.1)?

In this paper, by employing the mountain pass type theorem 1.4, for the case of $f\left(n, u_{n}\right)$ is neither superlinear nor sublinear, we will derive the new existence of two nontrivial periodic solutions for second-order discrete system (1.1), and our result is the following.

Theorem 1.5 Let $F \in C^{1}(R \times R, R)$ and for every $n \in Z, F(n, x)$ is twice continuously differentiable in $x$. Suppose that there is a positive integer $M \geq 3$ satisfying condition $\left(\mathrm{A}_{3}\right)$ and the following conditions:

$\left(W_{1}\right)$ for every $(n, x) \in Z \times R, F(n+M, x)=F(n, x)$;

$\left(W_{2}\right) F(n, 0)=0, \forall n \in Z$,

hold. Then system (1.1) has at least two nontrivial M-periodic solutions.

Remark 1.2 For the case of $f\left(n, u_{n}\right)$ is neither superlinear nor sublinear at $u_{n}$, by using linking theorem in [15], Zhou-Yu-Guo [1] obtained two nontrivial $M$-periodic solutions for system (1.1). Here, by using a different philosophy, i.e. an extension of the mountain pass type theorem, we also obtain the existence of two nontrivial $M$-periodic solutions.

Remark 1.3 Theorem 1.5 is concerned with $f\left(n, u_{n}\right)$ which is neither superlinear nor sublinear, and under condition $\left(\mathrm{W}_{1}\right)$ of Theorem 1.5 , we do not need $F \geq 0$.

Remark 1.4 We only need $F(n, 0)=0, \forall n \in Z$ in Theorem $1.5\left(\mathrm{~W}_{2}\right)$, which is also weaker than condition $\left(\mathrm{A}_{2}\right)$.

Example 1.2 Let $F(t, x)=a\left(x^{2} / 2+x+\cos x-1\right)(\phi(t)+K)$ with $x \in R, K>0$ such that the constant $a$ and the positive integer $M \geq 3$ satisfy

$$
\begin{cases}a>2, & \text { when } M \text { is even, } \\ a>2\left(1+\cos \frac{\pi}{M}\right), & \text { when } M \text { is odd. }\end{cases}
$$

Let $\phi(t) \in C^{1}(R, R)$ so that $\phi(t)$ is a $M$-periodic function satisfying $|\phi(t)|<K$. Then all assumptions in Theorem 1.5 are satisfied. Thus (1.1) has at least two nontrivial $M$-periodic solutions.

Example 1.3 Let $F(t, x)=a\left(\mu x^{2}+x+\cos x-1\right)(\phi(t)+K)$ with $x \in R, \mu \geq 1 / 2, K>0$, such that the constant $a$ and the positive integer $M \geq 3$ satisfy

$$
\begin{cases}a>2, & \text { when } M \text { is even, } \\ a>2\left(1+\cos \frac{\pi}{M}\right), & \text { when } M \text { is odd. }\end{cases}
$$


$\phi(t) \in C^{1}(R, R)$, and $\phi(t)$ is a $M$-periodic function satisfying $|\phi(t)|<K$. Then $F$ satisfies all assumptions in Theorem 1.5. Therefore, (1.1) has at least two nontrivial $M$-periodic solutions.

Remark 1.5 Obviously, $F$ in Examples 1.1, 1.2 and 1.3 satisfy all the assumptions in Theorem 1.5, but in Examples 1.2 and 1.3, $F$ satisfies neither condition $\left(\mathrm{A}_{1}\right)$ nor condition $\left(\mathrm{A}_{2}\right)$.

The paper is organized as follows: Sect. 2 is devoted to establishing a new quantitative deformation lemma. In Sect. 3, by using the new quantitative deformation lemma, we derive our new mountain pass theorem (Theorem 1.4). In Sect. 4, as an application of our new mountain pass theorem, we prove Theorem 1.5.

\section{New quantitative deformation lemma}

Lemma 2.1 Let $X$ be a Hilbert space and $\varepsilon$ be a small enough positive number. Let $\varphi \in$ $C^{2}(X, R), h \in R$. Assume that

$$
\left\|\varphi^{\prime}(u)\right\| \geq 2 \varepsilon, \quad \forall u \in \varphi^{-1}([h-2 \varepsilon, h+2 \varepsilon]) .
$$

Then there exists $\eta \in C(X, X)$, such that

(i) $\eta(u)=u, \forall u \notin \varphi^{-1}([h-2 \varepsilon, h+2 \varepsilon]) \backslash D$, where $D$ is any subset of $X$ satisfying $D \subset \varphi^{-1}\left(\left[h-\frac{1}{3} \varepsilon^{2}, h+\frac{1}{3} \varepsilon^{2}\right]\right)$

(ii) $\eta\left(\varphi^{-1}\left[h+\frac{1}{2} \varepsilon^{2}, h+\varepsilon^{2}\right]\right) \subset \varphi^{-1}\left(\left[h-\frac{3}{2} \varepsilon^{2}, h-\frac{1}{2} \varepsilon^{2}\right]\right)$.

Proof Let us define

$$
\begin{aligned}
& A:=\varphi^{-1}([h-2 \varepsilon, h+2 \varepsilon]) \backslash D, \quad B:=\varphi^{-1}\left(\left[h-\varepsilon, h-\frac{1}{2} \varepsilon\right]\right), \\
& C:=\varphi^{-1}\left(\left[h+\frac{1}{2} \varepsilon^{2}, h+\varepsilon^{2}\right]\right), \\
& \psi(u):=\frac{[\operatorname{dist}(u, C)-\operatorname{dist}(u, B)] \operatorname{dist}(u, X \backslash A)}{[\operatorname{dist}(u, C)+\operatorname{dist}(u, B)] \operatorname{dist}(u, X \backslash A)+\operatorname{dist}(u, B) \operatorname{dist}(u, C)} .
\end{aligned}
$$

Then $\psi$ is a locally Lipschitz continuous function such that

$$
\psi(u):= \begin{cases}1, & u \in B, \\ -1, & u \in C, \\ 0, & u \in X \backslash A .\end{cases}
$$

Let us also define a locally Lipschitz continuous vector field

$$
f(u):= \begin{cases}\psi(u)\left\|\varphi^{\prime}(u)\right\|^{-2} \varphi^{\prime}(u), & u \in A, \\ 0, & u \in X \backslash A .\end{cases}
$$


It is clear that $\|f(u)\| \leq(2 \varepsilon)^{-1}$ on $X$. For each $u \in X$, the Cauchy problem

$$
\left\{\begin{array}{l}
\frac{d}{d t} \sigma(t, u)=f(\sigma(t, u)) \\
\sigma(0, u)=u
\end{array}\right.
$$

has a unique solution $\sigma(\cdot, u)$ defined on $R$. Moreover, $\sigma$ is continuous on $R \times X$ (see e.g. [7]). By (2.2) and (2.3), then

$$
\begin{aligned}
\frac{d}{d t} \varphi(\sigma(t, u)) & =\left(\varphi_{\sigma}^{\prime}(\sigma(t, u)), \frac{d}{d t} \sigma(t, u)\right)=\left(\varphi_{\sigma}^{\prime}(\sigma(t, u)), f(\sigma(t, u))\right) \\
& =\psi(\sigma(t, u)) .
\end{aligned}
$$

On the other hand, with the help of (2.1), $\psi=0$ on $X \backslash A$, so the map $\eta$ defined on $X$ by $\eta(u):=\sigma\left(2 \varepsilon^{2}, u\right)$ satisfies $(\mathrm{i})$.

By (2.3), we have

$$
\sigma(t, u)=\sigma(0, u)+\int_{0}^{t} f(\sigma(s, u)) d s=u+\int_{0}^{t} f(\sigma(s, u)) d s, \quad \forall t \in\left[0,2 \varepsilon^{2}\right]
$$

Combining $\|f(u)\| \leq(2 \varepsilon)^{-1}$, we know

$$
\begin{aligned}
\|\sigma(t, u)-u\| & =\left\|\int_{0}^{t} f(\sigma(s, u)) d s\right\| \\
& \leq \int_{0}^{t}\|f(\sigma(s, u))\| d s \leq 2 \varepsilon^{2} \times \frac{1}{2 \varepsilon}=\varepsilon .
\end{aligned}
$$

Let $u \in \varphi^{-1}\left(\left[h+\frac{1}{2} \varepsilon^{2}, h+\varepsilon^{2}\right]\right)=C$, then $\psi(\sigma(0, u))=\psi(u)=-1$. Clearly, $\sigma(\cdot, u)$ is continuous on $R, \psi$ is locally Lipschitz continuous on $\sigma$, and $\psi(\sigma(0, u))=-1$ for $u \in C$. Thus, by (2.5), for $\varepsilon$ is small enough, we have

$$
\psi(\sigma(t, u)) \leq-\frac{3}{4}, \quad \forall t \in\left[0,2 \varepsilon^{2}\right]
$$

Therefore, it follows from (2.5) that

$$
\begin{aligned}
\varphi\left(\sigma\left(2 \varepsilon^{2}, u\right)\right) & =\varphi(u)+\int_{0}^{2 \varepsilon^{2}} \frac{d}{d t} \varphi(\sigma(t, u)) d t=\varphi(u)+\int_{0}^{2 \varepsilon^{2}} \psi(\sigma(t, u)) d t \\
& \leq h+\varepsilon^{2}+\left(-\frac{3}{4}\right) \times 2 \varepsilon^{2}=h-\frac{1}{2} \varepsilon^{2}
\end{aligned}
$$

and

$$
\begin{aligned}
\varphi\left(\sigma\left(2 \varepsilon^{2}, u\right)\right) & =\varphi(u)+\int_{0}^{2 \varepsilon^{2}} \frac{d}{d t} \varphi(\sigma(t, u)) d t=\varphi(u)+\int_{0}^{2 \varepsilon^{2}} \psi(\sigma(t, u)) d t \\
& \geq h+\frac{1}{2} \varepsilon^{2}-2 \varepsilon^{2}=h-\frac{3}{2} \varepsilon^{2} .
\end{aligned}
$$

So, (ii) is proved. 
Remark 2.1 The conclusion (ii) in Lemma 2.1 is different from conclusion (ii) in in Lemma 1.1.

Now, by Lemma 2.1, we can prove our mountain pass type theorem which is independent of $c_{0}$.

\section{Proof of Theorem 1.4}

Conclusion (i) is obvious. Suppose that conclusion (ii) does not hold. Consider $\beta=\eta \circ \gamma$, where $\eta$ is given by Lemma 2.1, and then we need to check two cases.

Case 1. $\varphi(0)<\varphi(e)=\varphi\left(e_{1}\right)<\hat{c}$.

By an analogous argument of conclusion (i) of Lemma 2.1, we have

$$
\begin{aligned}
& \beta(0)=\eta(\gamma(0))=\eta(0)=0, \quad \beta\left(\frac{1}{2}\right)=\eta\left(\gamma\left(\frac{1}{2}\right)\right)=\eta\left(e_{1}\right)=e_{1}, \\
& \beta(1)=\eta(\gamma(1))=\eta(e)=e .
\end{aligned}
$$

So, $\beta \in \hat{\Gamma}$. By the definition of $\hat{c}$, there exists $\gamma \in \hat{\Gamma}$ such that

$$
\max _{t \in[0,1]} \varphi(\gamma(t)) \leq \hat{c}+\varepsilon
$$

Then, from conclusion (ii) of Lemma 2.1 and (3.1), we have $\hat{c} \leq \max _{t \in[0,1]} \varphi(\beta(t)) \leq \hat{c}-\varepsilon$. This is a contradiction.

Case 2. $\varphi(0)<\varphi(e)=\varphi\left(e_{1}\right)=\hat{c}$.

Let us assume that $\max _{t \in[0,1]} \varphi(\gamma(t)) \equiv \hat{c}$ for every $\gamma \in \hat{\Gamma}$. Since $\varphi\left(e_{1}\right)=\hat{c}$ for every $0<\left\|e_{1}\right\|<r$ and $\varphi \in C^{2}(X, R)$, we see that conclusion (ii) holds. Thus, in the following discussion, we only need to argue the case of $\max _{t \in[0,1]} \varphi(\gamma(t)) \not \equiv \hat{c}$. Observing that $\max _{t \in[0,1]} \varphi(\gamma(t)) \geq \varphi\left(e_{1}\right)=\hat{c}$, there exists $\gamma_{1} \in \hat{\Gamma}$ such that $\max _{t \in[0,1]} \varphi\left(\gamma_{1}(t)\right)>\varphi\left(e_{1}\right)$. Note that $\gamma_{1}\left(\frac{1}{2}\right)=e_{1}, \varphi\left(e_{1}\right)=\hat{c}\left(0<\left\|e_{1}\right\|<r\right)$, and $\gamma_{1}(t) \in C([0,1], X)$ and $\varphi \in C^{2}(X, R)$, there exists $t_{1} \in[0,1]$ such that

$$
\left.\hat{c}+\frac{1}{2} \varepsilon^{2} \leq \varphi\left(\gamma_{1}\left(t_{1}\right)\right)\right) \leq \hat{c}+\varepsilon^{2} .
$$

Take $D=\{u \in X \mid h=\varphi(u)=\hat{c}\}$ in Lemma 2.1. Then, by $\varphi(0)<\varphi(e)=\varphi\left(e_{1}\right)=\hat{c}$ and conclusion (i) of Lemma 2.1, we have

$$
\begin{aligned}
& \beta(0)=\eta(\gamma(0))=\eta(0)=0, \quad \beta\left(\frac{1}{2}\right)=\eta\left(\gamma\left(\frac{1}{2}\right)\right)=\eta\left(e_{1}\right)=e_{1}, \\
& \beta(1)=\eta(\gamma(1))=\eta(e)=e .
\end{aligned}
$$

So, $\beta \in \hat{\Gamma}$. It follows from (3.2) and the conclusion (ii) of Lemma 2.1 that $\max _{t \in[0,1]} \varphi(\beta(t)) \leq \hat{c}-\frac{1}{2} \varepsilon^{2}$. Combining with $\beta(1)=e$, we must have $\max _{t \in[0,1]} \varphi(\beta(t)) \geq$ $\varphi(e)=\hat{c}$, which implies a contradiction.

Combining Case 1 and Case 2, the proof for our new mountain pass theorem is complete. 


\section{Proof of Theorem 1.5}

We divide the proof into eight steps.

Step 1: We introduce some notations.

- For $a, b \in Z$, define $Z[a]=\{a, a+1, \ldots\}, Z[a, b]=\{a, a+1, \ldots, b\}$ for $a \leq b$.

- Let the set of sequences $S=\left\{u=\left\{u_{n}\right\}=\left(\ldots, u_{-n}, \ldots, u_{0}, \ldots, u_{n}, \ldots\right), u_{n} \in R, n \in Z\right\}$. For any given positive integer $M, E_{M}$ is defined as a subspace of $S$ by

$$
E_{M}=\left\{u=\left\{u_{n}\right\} \in S \mid u_{n+M}=u_{n}, n \in Z\right\} .
$$

- For $x, y \in S, a, b \in R, a x+b y$ is defined by

$$
a x+b y=\left\{a x_{n}+b y_{n}\right\}_{n=-\infty}^{+\infty}
$$

then $S$ is a vector space. Clearly, $E_{M}$ is isomorphic to $R^{M}, E_{M}$ can be equipped with the inner product

$$
\langle x, y\rangle_{E_{M}}=\sum_{s=1}^{M} x_{s} y_{s}, \quad \forall x, y \in E_{M}
$$

then $E_{M}$ with the inner product given above is a finite dimensional Hilbert space and linearly homeomorphic to $R^{M}$. And we denote the norm $\|x\|=\left(\sum_{j=1}^{M} x_{j}^{2}\right)^{\frac{1}{2}}$.

- For a given matrix

$$
B=\left(\begin{array}{cccccc}
2 & -1 & 0 & \cdots & 0 & -1 \\
-1 & 2 & -1 & \cdots & 0 & 0 \\
0 & -1 & 2 & \cdots & 0 & 0 \\
\vdots & \vdots & \ddots & \vdots & \vdots & \vdots \\
-1 & 0 & 0 & \cdots & -1 & 2
\end{array}\right)_{M \times M}
$$

in view of the results established in [1], all the eigenvalues of $B$ are $0, \lambda_{1}, \lambda_{2}, \ldots, \lambda_{M-1}$ and $\lambda_{j}>0$ for all $j \in Z[1, M-1]$. Moreover,

$$
\lambda_{\min }=2\left(1-\cos \frac{2 \pi}{M}\right), \quad \lambda_{\max }= \begin{cases}4, & \text { when } M \text { is even, } \\ 2\left(1+\cos \frac{\pi}{M}\right), & \text { when } M \text { is odd }\end{cases}
$$

Step 2: Let the functional

$$
\varphi(u)=\frac{1}{2} \sum_{s=1}^{M}\left(\Delta u_{s}\right)^{2}-F\left(n, u_{n}\right)-G,
$$

where

$$
G=G\left(u_{1}, u_{2}, \ldots, u_{n-1}, u_{n+1}, u_{n+2}, \ldots, u_{M}\right)=w_{3}\left[\sum_{s=1}^{n-1}\left|u_{s}\right|^{3}+\sum_{s=n+1}^{M}\left|u_{s}\right|^{3}\right] .
$$


According to condition $\left(\mathrm{A}_{3}\right)$, if we let

$$
w=\max \left\{\left|F(n, x)-w_{3} x^{2}+w_{2}\right|: n \in Z,|x| \leq w_{1}\right\}
$$

and $\tilde{w}=w+w_{2}$, then

$$
F(n, x) \geq w_{3}|x|^{2}-\tilde{w} .
$$

Combining with the fact

$$
\sum_{s=1}^{M}\left(\Delta u_{s}\right)^{2}=\sum_{s=1}^{M}\left(u_{s+1}-u_{s}\right)^{2}=\sum_{s=1}^{M}\left(2 u_{s}^{2}-2 u_{s} u_{s+1}\right)
$$

for all $u \in E_{M}$, there exists a constant $w^{\prime}>\tilde{w}$ such that

$$
\begin{aligned}
\varphi(u) & =\frac{1}{2}\left[\sum_{s=1}^{M}\left(\Delta u_{s}\right)^{2}\right]-F\left(n, u_{n}\right)-G \\
& \leq \frac{1}{2} \sum_{s=1}^{M}\left(2 u_{s}^{2}-2 u_{s} u_{s+1}\right)-w_{3} u_{n}^{2}+\tilde{w}-w_{3} \sum_{s=1}^{n-1}\left|u_{s}\right|^{3}-w_{3} \sum_{s=n+1}^{M}\left|u_{s}\right|^{3} \\
& \leq \frac{1}{2} \sum_{s=1}^{M}\left(2 u_{s}^{2}-2 u_{s} u_{s+1}\right)-w_{3} u_{n}^{2}+w^{\prime}-w_{3} \sum_{s=1}^{n-1} u_{s}^{2}-w_{3} \sum_{s=n+1}^{M} u_{s}^{2} \\
& =\frac{1}{2} u^{\top} B u-w_{3}\|u\|^{2}+w^{\prime} \\
& \leq \frac{1}{2} \lambda_{\max }\|u\|^{2}-w_{3}\|u\|^{2}+w^{\prime} \\
& =\left(\frac{1}{2} \lambda_{\max }-w_{3}\right)\|u\|^{2}+w^{\prime} .
\end{aligned}
$$

Notice that if $M$ is even then $w_{3} \in(2,+\infty)$, and if $M$ is odd, then $w_{3} \in\left(1+\cos \frac{\pi}{M},+\infty\right)$, and

$$
\lambda_{\max }= \begin{cases}4, & \text { when } M \text { is even, } \\ 2\left(1+\cos \frac{\pi}{M}\right), & \text { when } M \text { is odd, }\end{cases}
$$

we have $\lambda_{\max } / 2-w_{3}<0$, which implies that $\varphi(u) \leq w^{\prime}$. Therefore, $\varphi(u)$ is bounded from above on $E_{M}$.

Step 3: Set $\tilde{c}=\sup _{u \in E_{M}} \varphi(u)$. From $\lambda_{\max } / 2-w_{3}<0$ and

$$
\varphi(u) \leq\left(\frac{\lambda_{\max }}{2}-w_{3}\right)\|u\|^{2}+w^{\prime}
$$

we have $\varphi(u) \rightarrow-\infty$ as $\|u\| \rightarrow+\infty$, which implies $-\varphi(u) \rightarrow+\infty$ as $\|u\| \rightarrow+\infty$. Hence, for every $l>|\tilde{c}|$, there is a constant $P>0$ such that, for every $\|u\|>P,-\varphi(u)>l>\tilde{c}$. With the help of the continuity of $\varphi(u)$, there must be a point $\bar{u} \in E_{M}$ such that $\varphi(\bar{u})=\tilde{c}=$ $\sup _{u \in E_{M}} \varphi(u)$ and $\|u\| \leq P$. Therefore, $\bar{u}$ is a critical point of the functional $\varphi(u)$ on $E_{M}$ with the critical value $\tilde{c}$. 
Next, let us search for the second critical point of the functional $\varphi(u)$ on $E_{M}$.

Step 4: Let $u^{(k)} \in E_{M}$, for all $k \in N$, be such that $\left\{\varphi\left(u^{(k)}\right)\right\}$ is bounded. From Step 2, there exists $M_{1}>0$, such that

$$
-M_{1} \leq \varphi\left(u^{(k)}\right) \leq\left(\frac{1}{2} \lambda_{\max }-w_{3}\right)\left\|u^{(k)}\right\|^{2}+w^{\prime}
$$

which implies that

$$
\left\|u^{(k)}\right\|^{2} \leq\left(w_{3}-\frac{1}{2} \lambda_{\max }\right)^{-1}\left(w^{\prime}+M_{1}\right) .
$$

That is, $\left\{u^{(k)}\right\}$ is bounded in $E_{M}$. Since $E_{M}$ is finite dimensional, there exists a subsequence of $\left\{u^{(k)}\right\}$ (not labeled), which is convergent in $E_{M}$, so the (P.S.) condition is verified.

Step 5: Let $f(t)=t^{2}-w_{3} t^{3}$ for $t \in[0,+\infty]$. Then $f^{\prime}(t)=2 t-3 w_{3} t^{2}=t\left(2-3 w_{3} t\right)$. So $f$ is increase on $\left[0,2 /\left(3 w_{3}\right)\right]$ and decrease on $\left(2 /\left(3 w_{3}\right), 1 / w_{3}\right)$. Combining $f(0)=0$ and $f\left(1 / w_{3}\right)=$ 0 , there exist $\xi \in\left(0,2 /\left(3 w_{3}\right)\right)$ and $\zeta \in\left(2 /\left(3 w_{3}\right), 1 / w_{3}\right)$, such that $f(\xi)=f(\zeta)>0$.

Step 6: By (4.1) and condition $\left(\mathrm{W}_{2}\right)$, we have $\varphi(0)=0$. Take

$$
e=\left\{\begin{array}{l}
u_{n-1}=\xi \\
u_{i}=0, \quad i=1,2, \ldots, n-2, n, n+1, \ldots, M,
\end{array}\right.
$$

and

$$
e_{1}=\left\{\begin{array}{l}
u_{n-1}=\zeta \\
u_{i}=0, \quad i=1,2, \ldots, n-2, n, n+1, \ldots, M
\end{array}\right.
$$

Then it is easy to verify that

$$
\begin{aligned}
\varphi(e) & =\frac{1}{2}\left[\sum_{s=1}^{M}\left(\Delta u_{s}\right)^{2}\right]-F\left(n, u_{n}\right)-G \\
& =u_{n-1}^{2}-w_{3}\left|u_{n-1}\right|^{3}=\xi^{2}-w_{3} \xi^{3}=f(\xi),
\end{aligned}
$$

and

$$
\begin{aligned}
\varphi\left(e_{1}\right) & =\frac{1}{2}\left[\sum_{s=1}^{M}\left(\Delta u_{s}\right)^{2}\right]-F\left(n, u_{n}\right)-G \\
& =u_{n-1}^{2}-w_{3}\left|u_{n-1}\right|^{3}=\zeta^{2}-w_{3} \zeta^{3}=f(\zeta) .
\end{aligned}
$$

In view of the fact that $f(\xi)=f(\zeta)>0,\|e\|=\xi$ and $\left\|e_{1}\right\|=\zeta$, we have $\varphi(e)=\varphi\left(e_{1}\right)>0=\varphi(0)$ and $\|e\| \neq\left\|e_{1}\right\|$. Moreover, all the assumptions in Theorem 1.4 are satisfied. Noticing that $\varphi(u)$ satisfies the (P.S.) condition, then, by Remark 1.1, there exists a critical point $\hat{u}$ such that $\varphi(\hat{u})=\hat{c}(\hat{c}$ is given in Theorem 1.4).

Step 7: In order to obtain two critical points, we also need to prove that $\hat{u} \neq \bar{u}$. Since $\varphi(\hat{u})=\hat{c}$ and $\varphi(\bar{u})=\tilde{c}$, if we can prove $\hat{c} \neq \tilde{c}$, that also implies $\hat{u} \neq \bar{u}$. So in the following, 
we are ready to prove that $\hat{c} \neq \tilde{c}$. Since $u \in E_{M}$ and $E_{M}$ is linearly homeomorphic to $R^{M}$, in Theorem 1.4, we can take $X=E_{M}$ and construct

$$
\begin{aligned}
\gamma_{1}(t) & =\left(u_{1}, \ldots, u_{n-2}, u_{n-1}, u_{n}, u_{n+1}, \ldots, u_{M+1}\right) \\
& =\left(0, \ldots, 0, u_{n-1}, 0,0, \ldots, 0\right) \\
& =\left(0, \ldots, 0,(2 \zeta-4 \xi) t^{2}+(-\zeta+4 \xi) t, 0,0, \ldots, 0\right)
\end{aligned}
$$

where $u_{n-1}=(2 \zeta-4 \xi) t^{2}+(-\zeta+4 \xi) t, t \in[0,1]$. Obviously, $\gamma_{1} \in C([0,1])$.

One computes that $\gamma_{1}(0)=(0, \ldots, 0,0,0,0, \ldots, 0)$,

$$
\gamma_{1}\left(\frac{1}{2}\right)=\left(0, \ldots, 0, u_{n-1}, 0,0, \ldots, 0\right)=(0, \ldots, 0, \xi, 0,0, \ldots, 0)=e
$$

and

$$
\gamma_{1}(1)=\left(0, \ldots, 0, u_{n-1}, 0,0, \ldots, 0\right)=(0, \ldots, 0, \zeta, 0,0, \ldots, 0)=e_{1} .
$$

Hence, $\gamma_{1}(t) \in \hat{\Gamma}$ where

$$
\hat{\Gamma}:=\left\{\gamma \in C([0,1], X): \gamma(0)=0, \gamma\left(\frac{1}{2}\right)=e_{1}, \gamma(1)=e\right\} .
$$

Let $u=\gamma_{1}(t), t \in[0,1]$ be in (4.1). Since $F(n, 0)=0$, we have

$$
\begin{aligned}
\varphi(u) & =\varphi\left(\gamma_{1}(t)\right)=\frac{1}{2}\left[\sum_{s=1}^{M}\left(\Delta u_{s}\right)^{2}\right]-F\left(n, u_{n}\right)-G \\
& =\frac{1}{2}\left(u_{n-1}^{2}+u_{n-1}^{2}\right)-F(n, 0)-w_{3}\left|u_{n-1}\right|^{3} \\
& =u_{n-1}^{2}-w_{3}\left|u_{n-1}\right|^{3} .
\end{aligned}
$$

Let $\left|u_{n-1}\right|=y \in[0,+\infty]$, then, by (4.2), we see that when $y=2 / 3 w_{3}, \varphi\left(\gamma_{1}(t)\right)=y^{2}-w_{3} y^{3}$ takes the maximum value $4 /\left(27 w_{3}^{2}\right)$. We must point that when $t \in[0,1],\left|u_{n-1}\right|$ can take the value of $2 / 3 w_{3}$. In fact, when $t=0$,

$$
u_{n-1}=(2 \zeta-4 \xi) t^{2}+(-\zeta+4 \xi) t=0
$$

and when $t=1$,

$$
u_{n-1}=(2 \zeta-4 \xi) t^{2}+(-\zeta+4 \xi) t=\zeta
$$

Observing that $\zeta \in\left(2 /\left(3 w_{3}\right), 1 / w_{3}\right)$, then, by the continuity of $u_{n-1}=(2 \zeta-4 \xi) t^{2}+(-\zeta+4 \xi) t$, there exists $\tilde{t} \in[0,1]$ such that $u_{n-1}=(2 \zeta-4 \xi) \tilde{t}^{2}+(-\zeta+4 \xi) \tilde{t}=2 /\left(3 w_{3}\right)$.

Since $\hat{c}:=\inf _{\gamma \in \hat{\Gamma}} \max _{t \in[0,1]} \varphi(\gamma(t))$ and $\gamma_{1}(t) \in \hat{\Gamma}$, we have $\hat{c} \leq 4 /\left(27 w_{3}^{2}\right)$. In order to obtain two critical point of $\varphi(u)$ on $E_{M}$, we will show that $\tilde{c}>4 /\left(27 w_{3}^{2}\right)$.

Since $E_{M}$ is linearly homeomorphic to $R^{M}$ and $M \geq 3$, if we choose

$$
u=\left(u_{1}, \ldots, u_{n-2}, u_{n-1}, u_{n}, u_{n+1}, \ldots, u_{M+1}\right) \in E_{M},
$$


then

$$
u_{1}=\cdots=u_{n-2}=u_{n}=u_{n+2}=\cdots=u_{M}=0, \quad u_{n-1}=-u_{n+1}=-\frac{1}{2 w_{3}} .
$$

By $(4.1)$ and $F(n, 0)=0$, we have

$$
\begin{aligned}
\varphi(u) & =\frac{1}{2} \sum_{s=1}^{M}\left(\Delta u_{s}\right)^{2}-F\left(n, u_{n}\right)-G \\
& =\frac{1}{2}\left(u_{n-1}^{2}+u_{n-1}^{2}+u_{n+1}^{2}+u_{n+1}^{2}\right)-F(n, 0)-w_{3}\left(u_{n-1}^{3}+u_{n+1}^{3}\right) \\
& =1 /\left(2 w_{3}^{2}\right)-0-1 /\left(4 w_{3}^{2}\right)=1 /\left(4 w_{3}^{2}\right) .
\end{aligned}
$$

Employing $\tilde{c}=\sup _{u \in E_{M}}$, we have $\tilde{c} \geq 1 /\left(4 w_{3}^{2}\right)>4 /\left(27 w_{3}^{2}\right) \geq \hat{c}$. Combining $\varphi(\hat{u})=\hat{c}, \varphi(\bar{u})=\tilde{c}$, both $\hat{u}$ and $\bar{u}$ are critical point of the functional $\varphi$, we obtain two different critical points of $\varphi$.

Step 8: (1.1) has at least two nontrivial $M$-periodic solutions. Since $\varphi \in \mathcal{C}^{2}\left(E_{M}, R\right)$, for any $u=\left\{u_{n}\right\}_{n \in Z} \in E_{M}$, according to $u_{0}=u_{M}, u_{1}=u_{M+1}$, one computes that

$$
\frac{\partial \varphi}{\partial u_{n}}=\Delta^{2} u_{n-1}+\nabla_{u_{n}} F\left(n, u_{n}\right), \quad \forall n \in Z .
$$

Therefore, the existence of critical points of $\varphi$ on $E_{M}$ implies the existence of periodic solutions of system (1.1). Moreover, we obtained two different critical points of $\varphi(u)$ on $E_{M}$ in Step 7, so system (1.1) has two different $M$-periodic solutions.

Note that in (4.1), $\varphi(0)=0$. But $\varphi(\hat{u})=\hat{c} \geq \varphi(e)>\varphi(0)=0$ and $\varphi(\bar{u})=\tilde{c}=\sup _{u \in E_{M}}>$ $4 /\left(27 w_{3}^{2}\right) \geq \hat{c} \geq \varphi(e)>0$, so any of the above periodic solutions $\hat{u}$ and $\bar{u}$ is nontrivial. From this, Theorem 1.5 is proved.

Remark 4.1 Let $F(t, x)$ be stated in Example 1.2, from (4.1), we have

$$
\begin{aligned}
\varphi(u)= & \frac{1}{2}\left[\sum_{s=1}^{M}\left(\Delta u_{s}\right)^{2}\right]-a\left(u_{n}^{2} / 2+u_{n}+\cos u_{n}-1\right)(\phi(n)+K) \\
& -w_{3}\left[\sum_{s=1}^{n-1}\left|u_{s}\right|^{3}+\sum_{s=n+1}^{M}\left|u_{s}\right|^{3}\right],
\end{aligned}
$$

where $u=\left(\ldots, u_{-n}, \ldots, u_{0}, \ldots\right) \in E_{M}$. We notice that the value of $\inf _{\|u\|=r} \varphi(u)$ is very difficult to compute, but fortunately the condition in our new mountain pass theorem (Theorem 1.4) is independent of $\inf _{\|u\|=r} \varphi(u)$, and we need not compute it.

\section{Acknowledgements}

The authors express their sincere gratitude to the editors and referees for their helpful comments and suggestions.

\section{Funding}

The first author is partially supported by Graduate Science Innovation Foundation of Sichuan University (2018YJSY045). The second author is partially supported by National Science Foundation of China (11501577), and the third author is partially supported by National Science Foundation of China (116712787). 
Authors' contributions

The authors contributed equally to this paper. All authors read and approved the final manuscript.

\section{Author details}

${ }^{1}$ School of Data Science and Information Engineering, Guizhou Minzu University, Guiyang, China. ${ }^{2}$ School of Mathematics, Sichuan University, Chengdu, China. ${ }^{3}$ School of Statistics and Mathematics, Zhongnan University of Economics and Law, Wuhan, China.

\section{Publisher's Note}

Springer Nature remains neutral with regard to jurisdictional claims in published maps and institutional affiliations.

Received: 15 November 2018 Accepted: 14 April 2019 Published online: 23 April 2019

\section{References}

1. Zhou, Z., Yu, J.S., Guo, Z.M.: Periodic solutions of higher-dimensional discrete systems. Proc. R. Soc. Edinb. A 134 1013-1022 (2004)

2. Ambrosetti, A., Rabinowitz, P.H.: Dual variational methods in critical point theory and applications. J. Funct. Anal. 49 349-381 (1973)

3. Willem, M.: Minimax Theorems. Birkhäuser, Boston (1996)

4. Chang, K.C.: Infinite Dimensional Morse Theory and Multiple Solution Problems, vol. 6. Birkhäuser, Boston (1993)

5. Brezis, H., Coron, J.M., Nirenberg, L.: Free vibrations for a nonlinear wave equation and theorem of P. Rabinowitz. Commun. Pure Appl. Math. 33, 667-684 (2010)

6. Ghoussoub, N.: Duality and Perturbation Methods in Critical Point Theory. Cambridge University Press, Cambridge (1993)

7. Schwartz, L.: Cours d'analyse. Hermann, Paris (1991-1994)

8. Pucci, P., Serrin, J.: A mountain pass theorem. J. Differ. Equ. 60, 142-149 (1985)

9. Qi, G.J.: Extension of mountain pass lemma. Chin. Sci. Bull. 32, 798-801 (1987)

10. Chen, P., Fang, H.: Existence of periodic and subharmonic solutions for second-order $p$-Laplacian difference equations. Adv. Differ. Equ. 2007, 042530 (2007)

11. Deng, X.Q., Shi, H.P., Xie, X.L.: Periodic solutions of second order discrete Hamiltonian systems with potential indefinite in sign. Appl. Math. Comput. 218, 148-156 (2011)

12. Ding, L., Wei, J.L.: Notes on multiple periodic solutions for second-order discrete Hamiltonian system. Dyn. Syst. 32, 544-552 (2017)

13. Guo, Z.M., Yu, J.S.: The existence of periodic and subharmonic solutions for second-order superlinear difference equations. Sci. China Math. 46, 506-515 (2003)

14. Guo, Z.M., Yu, J.S.: The existence of periodic and subharmonic solutions to subquadratic second-order difference equations. J. Lond. Math. Soc. 68, 419-430 (2003)

15. Rabinowitz, P.H.: Minimax Methods in Critical Point Theory with Applications to Differential Equations, vol. 65. Am. Math. Soc., Providence (1986)

16. Xue, Y.F., Tang, C.L.: Existence of a periodic solution for subquadratic second-order discrete Hamiltonian system. Nonlinear Anal., Theory Methods Appl. 67, 2072-2080 (2007)

17. Xue, Y.F., Tang, C.L.: Multiple periodic solutions for superquadratic second-order discrete Hamiltonian systems. Appl. Math. Comput. 196, 494-500 (2008)

18. Yu, J.S., Deng, X.Q., Guo, Z.M.: Periodic solutions of a discrete Hamiltonian system with a change of sign in the potential. J. Math. Anal. Appl. 324, 1140-1151 (2006)

\section{Submit your manuscript to a SpringerOpen ${ }^{\circ}$ journal and benefit from:}

- Convenient online submission

- Rigorous peer review

- Open access: articles freely available online

- High visibility within the field

- Retaining the copyright to your article

Submit your next manuscript at $\boldsymbol{~ s p r i n g e r o p e n . c o m ~}$ 